Results The project is about to be launched and will be fully evaluated by patient outcomes, changes in patterns of attendance at $\mathrm{ED}$ and collecting patient narratives to look at trends in those using the integrated service.

Conclusion Working in partnership we hope to reduce emergency admissions and improve the pathway for patients.

\section{P-223 ALL ABOUT ME: THIS DOCUMENT WILL HELP US SUPPORT YOU IN AN UNFAMILIAR PLACE}

Jan Proud. St Margaret's Hospice, Taunton, UK

10.1136/bmjspcare-2017-hospice.248

'This document is intended to provide staff with information about the person being cared for as an individual. This will enhance the care and support given while patients are in an unfamiliar environment. It is not a medical document. It is about the person at the time the document is completed and may need to be updated if necessary. It can be completed by the person themselves or with help from their carer. It has been adapted from a similar document titled 'This is me' produced by the Royal College of Nursing and the Alzheimer's Society.' The 'This is me' document has been adapted to remove references to dementia and tailor the questions more to the individual needs of patients within the hospice environment. The idea was to get away from the electronic computer system of recording patient care and interactions, to have a document that represented the patient as an individual with individual wishes, hopes, fears and desires. The Hospice ethos is to have patient-centred, family-focused care and I believe this document encourages this from first contact with a family. Questions such as: 'The kind of things that help me feel at peace are...'; 'My home and family, things that are important to me...'; 'I like to relax by...' are specific questions which help our Spiritual Care Team form closer bonds with patients and their carers. Other prompts help clarify issues for members of our multi-disciplinary team such as questions regarding 'My Mobility' are designed to help assessments for the physiotherapy team. All of the questions have value to teams and the information is considered vital for holistic, patient-centred care. We also aim to help patients and relatives celebrate special events or wishes whenever possible as identified in this document.

\section{P-224 THEMATIC ANALYSIS OF REFERRAL CONTROL UPON THE NATURE OF COMMUNITY SERVICE RESPONSE VISITS}

Simon Edgecombe, Robb Clapp. St John's Hospice, Lancaster, UK

\subsection{6/bmjspcare-2017-hospice.249}

Aims To determine whether the introduction of refined referral limitations, including daily triage, influenced the nature of the response visits undertaken by the community 'Hospice at Home' team, with particular attention being paid to the type of care requested and provided and the condition of the patient.

Methods The research was conducted in the form of a thematic analysis (see Braun \& Clarke, 2006; Seal, 2016) using the community clinical records which documented the community service response visits. A sample of three groups was selected from patients who had requested and received response visits in June, September and December of 2016, with June acting as the pre-change control group. The encounter records were then fed through a predetermined coding matrix, focusing upon the source of the request, the reason for the request, the condition in which the attended patient was found to be in, the emotional state of the family or carers and the actions undertaken.

Results It was found that while the introduction of refined referral parameters and daily triage meetings did not influence the need for supplementary tasks, such as personal care or psychological support for the patient or family/carers, or the documented levels of distress amongst family or carers, several transitional themes became apparent. Increased restrictions to referral acceptance amplified the ratio presence of patients who were at the very end of life, with activities subsequently becoming much more focused upon those associated with end of life care and reducing the presence of actions less associated with palliative conditions.

Conclusions The evidence compiled suggests that, whilst more refined referral criteria may at first assessment risk a detrimental impact upon response visits in the community, greater control over the referral process helps with prioritisation and improves responsiveness towards patients with more urgent palliative needs.

\section{P-225 INNOVATING A WHOLE TEAM APPROACH TO COMMUNITY PALLIATIVE CARE}

Gaberielle Linehan, Caroline Betts. St Raphael's Hospice, Sutton, UK

\subsection{6/bmjspcare-2017-hospice.250}

Background How often have you heard comments from colleagues about time pressures and unmanageable workloads? In order to redress this situation with a focus on effectiveness, efficiency and quality we took the decision to innovate the way in which our Community Palliative Care Team (CPCT) delivered supportive end of life care.

Aims This is a work in progress, with the aim of becoming one team, facilitating further integration of approach in delivering and responding to patients and healthcare care professionals.

Methods A review of working hours was undertaken, facilitating long days as well as traditional 9-5 and annualised hours for one CNS; with 9-5 worked on call at weekends. Specific roles were designated to cover triage, routine telephone contacts and a CNS as emergency responder: all roles were shared. Visits are scheduled by CNSs across the team, on the days people are working. No individualised case loads. Initially, the service was split into two areas to embed the new working routine and allow for any issues to be identified. Electronic patient records and iIPads facilitate remote working negating the need to return to base across the day.

Results The new mode of operandi has had a significant impact across the community service. Team case loads have resulted in a $15 \%$ increase in routine visits across the day/evening, with improvement in responsiveness to urgent visits, average increases in telephone contacts with patients up $40 \%$ and to HCP up 19\%. The team self support and manage their work with a marked reduction in working late, absence and improvement in morale. A shared team approach has reduced patient dependency on individuals, patients are reassurred that 